\title{
Single-frequency Raman Distributed-feedback Fiber Laser
}

\author{
Jindan Shi, Shaif-ul Alam, Morten Ibsen \\ Optoelectronics Research Centre, University of Southampton, Highfield, Southampton, SO17 1BJ, UK \\ jxs@orc.soton.ac.uk
}

\begin{abstract}
We report a 30cm-long Raman Distributed-feedback laser with $\sim 450 \mathrm{~mW}$ threshold in a commercial Ge/Si fiber. The laser is pumped with up to $1.3 \mathrm{~W}-\mathrm{CW}$ at $1064 \mathrm{~nm}$ and exhibits singlefrequency performance with a linewidth $<2.5 \mathrm{kHz}$ at $1109.5 \mathrm{~nm}$.

OCIS codes: (060.3735) Fiber Bragg gratings; (140.3490) Lasers, distributed-feedback; (140.3550) Lasers, Raman; (140.3510) Lasers, fiber.
\end{abstract}

\section{Introduction}

Single-frequency, narrow linewidth and low noise fiber laser sources are intensively desirable for applications including high-resolution interferometry, sensing, coherent communications, dense WDM network systems, and LIDAR. These performance features have been achieved in heavily rare-earth (RE)-doped fibers with unidirectional ring configurations [1], short-cavity distributed Bragg reflector (DBR) designs [2] and distributed-feedback (DFB) structures [3-5]. However, the lasing wavelength region of RE-doped lasers is naturally limited by the specific RE ions used. Instead, a Raman gain-based DFB fiber laser is of considerable interest for overcoming not just the limitations in wavelength coverage from its rare-earth doped counterparts, but also because it opens up the prospect of generating high-power narrow linewidth low-noise oscillation at, in principle, any desired wavelength ranging from the visible to the infrared region [6-8]. In contrast to what typically can be achieved from rare-earth gain, the Raman gain coefficient of silica fiber is typically low. Thus, watt-level of pump powers and long lengths of (>10 cms) DFB gratings have been highlighted as being required for efficient lasing [9]. R-DFB fiber lasers at $1.6 \mu \mathrm{m}$ have been demonstrated in commercial Raman fiber and highly nonlinear fiber with thresholds of $39 \mathrm{~W}$ and $4.3 \mathrm{~W}$, respectively [7]. The linewidth in that particular work was reported to be in the $\mathrm{MHz}$ region. Previously, we have demonstrated R-DFB fiber lasers at $1.1 \mu \mathrm{m}$ with a threshold value as low as $\sim 1 \mathrm{~W}$, high efficiency (up to $92 \%$ ) and high output power (up to $2 \mathrm{~W}$ ) in $30 \mathrm{cms}$ long centre $\pi$ phase-shifted DFB gratings in commercial Ge/Si fibers [8]. The pump used in that work was an unpolarized $1.06 \mu \mathrm{m}$ Yb-doped fibre MOPA laser system. In this work, we report a further improvement/reduction of the threshold power by using a linearly polarized fiber MOPA laser at $1.06 \mu \mathrm{m}$. From the same R-DFB sample as the one used in [9], the pump threshold has been reduced by a factor of 2 [10]. The demonstrated threshold power is $\sim 450 \mathrm{~mW}$ with an output power of $70 \mathrm{~mW}$ for $1.3 \mathrm{~W}$ of incident pump power. Moreover, we demonstrate that this R-DFB fiber laser exhibits single-frequency performance at $1109.5 \mathrm{~nm}$ with a linewidth measured to be less than $2.5 \mathrm{kHz}$.

\section{Experiment}

The schematic diagram of the experimental setup is shown in Fig.1(a). A continous-wave (CW), linearly polarized, $\mathrm{Yb}$-doped PM fiber MOPA at $1.06 \mu \mathrm{m}$ is used as the pump source. The output is spliced to a non-PM high power isolator and a polarization controller (PC), which is used to align the pump polarization state to the fiber DFB grating. $1064 \mathrm{~nm} / 1117 \mathrm{~nm}$ wavelength division multiplexers (WDM1/2/3) are used to separate the R-DFB signal from the pump both at the backward and forward output ends of the DFB grating. All fiber ends are angle-cleaved to prevent end-feedback, and all components (WDMs and DFB gratings) are mounted on heat sinks to help control the temperature and better remove any generated heat.
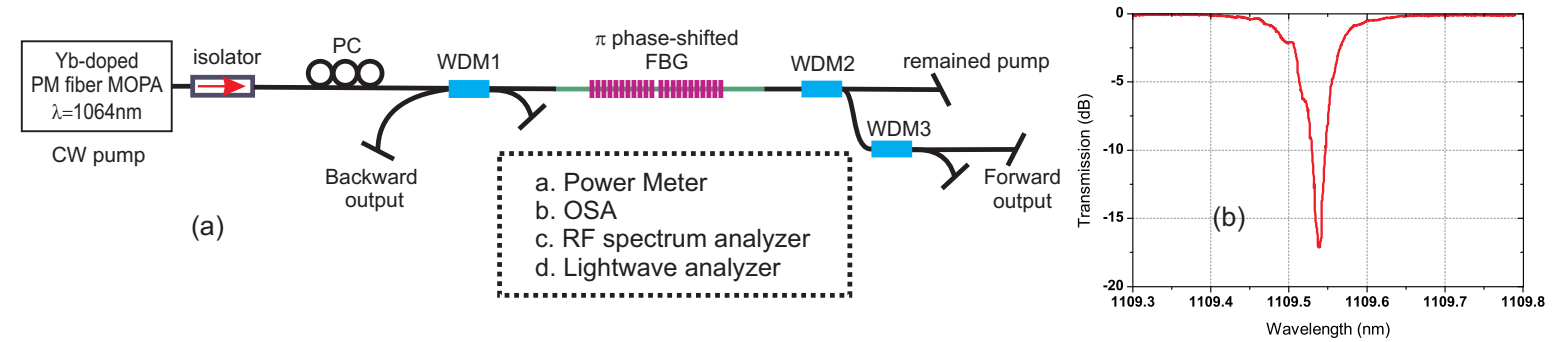

Fig.1 (a) Schematic diagram of experimental setup. Dotted box denotes the measurement equipment used to characterize the R-DFB fiber laser. (b) Transmission spectrum of the DFB grating with $0.01 \mathrm{~nm}$ resolution bandwidth (RBW).

A $30 \mathrm{~cm}$-long centre $\pi$ phase-shifted DFB grating has been written in a commercial high germanium doped silica fiber (UHNA4 from Nufern), using a 244nm CW UV-laser [11]. Fig.1 (b) shows the transmission spectrum of the 
grating. The index modulation is measured to be $\sim 10^{-5}$ and the grating inscription-induced loss has previously been found to be negligible [8]. The NA, mode field diameter (MFD), cut-off wavelength and background loss of the fiber is $0.35,2.6 \pm 0.3 \mu \mathrm{m}$ (@1100nm), $1050 \pm 50 \mathrm{~nm}$ and $\sim 5 \mathrm{~dB} / \mathrm{km}$, respectively.
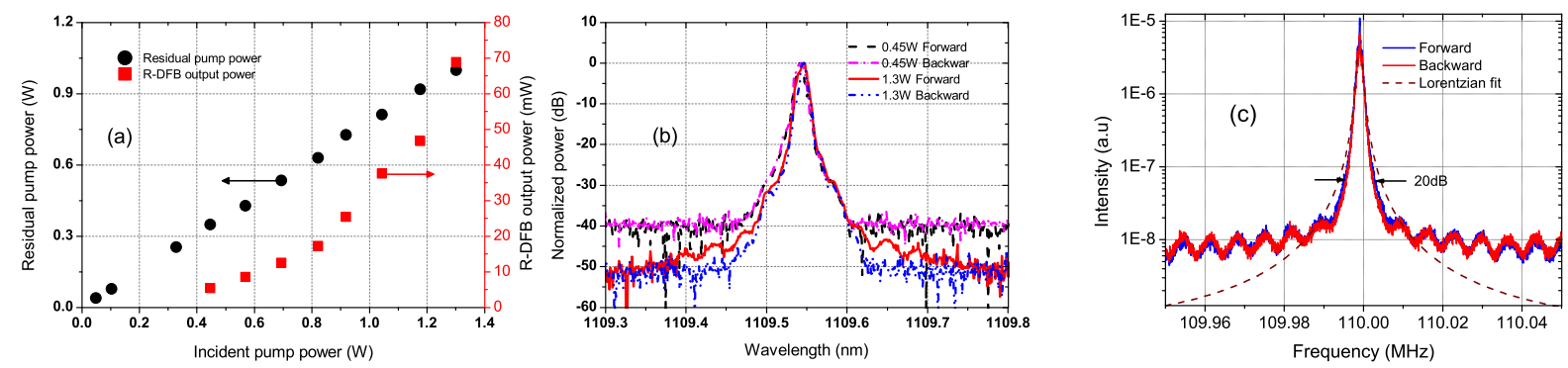

Fig.2 (a) R-DFB total output power and residual pump power against the incident pump power. (b) R-DFB output spectra (0.01nm RBW) at the threshold and the maximum pump power in this experiment, respectively. The inset power indicates the incident pump power. (c) Delayed self-heterodyne RF-spectra of the forward and backward output of the R-DFB.

\section{Results and discussions}

Fig.2 (a) shows the total R-DFB output power [ $[$ ] and residual pump power [ $\bullet$ ] against the incident pump power. The threshold is observed to be only $\sim 450 \mathrm{~mW}$, and the output power is seen to grow nonlinearly with increasing incident pump power. This behavior is similar to what we observed previously [9]. Fig.2 (b) shows the R-DFB spectra measured at the threshold and maximum pump power with an Advantest optical spectrum analyzer (Q8384) at a resolution of $0.01 \mathrm{~nm}$. The signal-to-noise $(\mathrm{SNR})$ ratio is maintained above $40 \mathrm{~dB}$ and the R-DFB signal wavelength is found to be constant for all the pump powers. This indicates that thermal effects on the R-DFB is negligible. A more accurate linewidth measurement is carried out with a delayed self-heterodyne interferometer with a $29.75 \mathrm{~km}$ delay line. The nominal resolution of this setup is $2.5 \mathrm{kHz}$ at the R-DFB signal wavelength. Fig.2 (c) shows the heterodyne RF-spectra of the R-DFB fiber laser at $\sim 9 \mathrm{~mW}$ output power for forward and backward signals respectively, with the noise bandwidth of $39.14 \mathrm{~Hz}$. Due to the negligible thermal effects in the laser, a Lorentziancurve is fitted to the experimental data with a resulting FWHM of only $\sim 1.2 \mathrm{kHz}$. The effects of the long coherence length of the R-DFB laser are obvious from the distinguishable sidelobes of the heterodyne spectra. Thus, the true linewidth of the R-DFB laser is believed to be less than $2.5 \mathrm{kHz}$. We also examined the heterodyne RF-spectra in a wide frequency span from $10 \mathrm{MHz}$ to $5 \mathrm{GHz}$. Any side modes or higher longitudinal-modes should show up as a beat signal with the main mode beat signal at $110 \mathrm{MHz}$ (the AOM shift frequency). However, no such beat signals were observed down to the noise floor. Hence, we can deduce that the R-DFB fiber laser is indeed a single-frequency laser. The R-DFB signal polarization extinction ratio (PER) is measured to be $>13 \mathrm{~dB}$.

\section{Conclusions}

In summary, we have demonstrated a narrow-linewidth $(<2.5 \mathrm{kHz})$, low-threshold $(\sim 450 \mathrm{~mW})$ Raman DFB fiber laser emitting at $1109.5 \mathrm{~nm}$. To the best of our knowledge, this is the narrowest linewidth and lowest threshold ever reported from a short-length Raman fiber laser.

\section{References}

[1] K. Iwatsuki, H. Okamura, and M. Saruwatari, "Wavelength-tunable single-frequency and single-polarization Er-doped fiber ring laser with 1.4 kHz linewidth," Electron. Lett., 26, 2033-2034 (1990).

[2] C. Spiegelberg, J. Geng, Y. Hu, Y. Kaneda, S. Jiang, and N. Peyghambarian, "Low-Noise Narrow-Linewidth Fiber Laser at 1550 nm (June 2003)," J. Lightwave Technol., 22, 57-62 (2004).

[3] W. H. Loh and R. I. Laming, "1.55 $\mu \mathrm{m}$ phase-shifted distributed feedback fiber laser," Electron. Lett., 31, 1440-1442 (1995).

[4] L. B. Fu, M. Ibsen, D. J. Richardson, and D. N. Payne, "977-nm all-fiber DFB laser," Photon. Technol. Lett., 16, 2442-2444 (2004).

[5] N. Y. Voo, J. K. Sahu, and M. Ibsen, "345-mW 1836-nm single-frequency DFB fiber laser MOPA," Photon. Technol. Lett., 17, 2550-2552 (2005).

[6] V. E. Perlin and H. G. Winful, "Distributed feedback fiber Raman laser," J. Quantum. Elec., 37, 38-47 (2001).

[7] P. S. Westbrook, K. S. Abedin, J. W. Nicholson, T. Kremp, and J. Porque, "Raman fiber distributed feedback lasers," Opt. Lett., 36, 28952897 (2011).

[8] J. Shi, S.-u. Alam, and M. Ibsen, "High power, low threshold, Raman DFB fiber lasers," in proceedings to IQEC/CLEO Pacific Rim Sydney, postdeadline paper (2011).

[9] J. Shi, S.-u. Alam, and M. Ibsen, "Highly Efficient Raman Distributed Feedback Fiber Lasers", submitted to Optics Express (2011)

[10] G. P. Agrawal, "Nonlinear fiber optics," second edition (1995).

[11] M. Ibsen, M.K. Durkin, M.J. Cole and R.I. Laming, "Sinc-sampled fiber Bragg gratings for identical multiple wavelength operation",

Photon. Technol. Lett., 10, 842-844 (1998). 